DOI: 10.12957/demetra.2018.30654

\title{
Estado nutricional e transtornos do comportamento alimentar em estudantes do curso de graduação em nutrição do Instituto Federal de Educação, Ciência e Tecnologia, Ceará, Brasil
}

\author{
Nutritional status and eating disorders among students of the nutrition undergraduate course \\ at the Instituto Federal de Educação, Ciência e Tecnologia, Ceará state, Brazil
}

\author{
Raimunda Gerlane Lima Maia \\ Bárbara de Cerqueira Fiorio² \\ Juliana Zani de Almeida' \\ Francisco Regis da Silva ${ }^{3}$ \\ I Instituto Federal de Educação, Ciência e \\ Tecnologia do Ceará, Curso de Nutrição. Limoeiro \\ do Norte, CE, Brasil. \\ ${ }^{2}$ Instituto Federal de Educação, Ciência e \\ Tecnologia do Ceará, Unidade de Alimentação e \\ Nutrição. Fortaleza, CE, Brasil. \\ ${ }^{3}$ Universidade Estadual do Ceará, Centro de \\ Ciências da Saúde, Programa de Pós-graduação \\ em Saúde Coletiva. Fortaleza, CE, Brasil. \\ Correspondência / Correspondence \\ Francisco Regis da Silva \\ E-mail: regisfrs@hotmail.com
}

\section{Resumo}

Transtornos alimentares tendem a ocorrer entre mulheres jovens, colocando universitárias como grupo de risco. Este estudo objetivou avaliar o estado nutricional agregado à percepção da imagem corporal e ao comportamento alimentar de estudantes do sexo feminino do curso de Nutrição. Trata-se de estudo transversal, do tipo descritivo e analítico, com amostra aleatória representativa, que utilizou o índice de massa corporal (IMC) autorreferido; o Body Shape Questionnaire (BSQ); o Bulimic Investigatory Test Edinburg (BITE); e o Eating Attitudes Test (EAT26). Foram empregadas análises descritivas de média \pm desvio padrão (DP) e frequências simples e relativas. Para a análise de diferenças estatísticas, utilizou-se a análise de variância (Anova), e o nível de significância entre os grupos foi determinado pelo teste de Turkey, com nível de significância $\mathrm{p}<0,05$. As médias \pm DP de idade e IMC foram $23 \pm 4$ anos e 23,39 \pm 4,01, respectivamente. As prevalências foram: $46,14 \%$ apresentaram distorção da imagem corporal pelo BSQ; 9,62\% tiveram risco de desenvolver distúrbios alimentares pelo EAT-26; e 15,38\% expressaram comportamento alimentar anormal. Na população estudada, há grande preocupação com o corpo, o que demonstra influência da pressão sociocultural nesse grupo.

Palavras-chave: Transtornos da Alimentação e da Ingestão de Alimentos. Imagem Corporal. Estado Nutricional. 


\section{Abstract}

Eating disorders tend to occur among young women, placing university students as a risk group. This study aimed to evaluate the nutritional status associated to the perception of the body image and the eating behavior of female students in a Nutrition course. It is a cross-sectional, descriptive and analytical study with a representative random sample, using the self-reported body mass index (BMI); Body Shape Questionnaire (BSQ); the Bulimic Investigatory Test Edinburg (Bite); and the Eating Attitudes Test (EAT-26). Descriptive analyzes of mean \pm standard deviation (SD) and simple and relative frequencies were used. Statistical analysis was used to analyze variance (ANOVA) and significance level between the groups was determined by the Turkey test, with a significance level of $\mathrm{p}<0.05$. The means \pm SD of age and BMI were $23 \pm 4$ years and $23.39 \pm 4.01$, respectively. Prevalence were: $46.14 \%$ reveal distortion of the body image by BSQ; 9.62\% had a risk of developing eating disorders in EAT-26 and $15.38 \%$ expressed abnormal eating behavior. In the studied population there is great concern about the body, demonstrating the influence of sociocultural pressure on this group.

Keywords: Eating disorders and Food Intake. Body Image. Nutritional Status.

\section{Introdução}

Os transtornos alimentares (TAs) são transtornos psiquiátricos, e seus portadores se caracterizam por apresentarem desvio de padrão no comportamento alimentar e distorção da imagem corporal, que causam severo prejuízo a sua saúde, como: alterações físicas, endócrinas, pulmonares, renais, cardíacas, hematológicas, hidroeletrolíticas e ósseas, complicações metabólicas, entre outros. São patologias que geralmente apresentam suas primeiras manifestações na infância e adolescência. ${ }^{1}$ Os principais tipos de TAs são a anorexia nervosa (AN) e a bulimia nervosa (BN). ${ }^{1}$

A AN é caracterizada por limitações na dieta, começando com uma redução de alimentos mais calóricos, progredido até um jejum total. ${ }^{2}$ A BN é caracterizada pela ingestão de uma grande quantidade de alimento em um intervalo de tempo pequeno, sendo que a percepção desse fato leva o paciente a medidas extremas, que são os chamados episódios bulímicos. Nesse momento, o indivíduo apresenta um grande sentimento de culpa e tenta compensar o ato feito através de vômitos provocados ou autoinduzidos e atividade física excessiva. ${ }^{3,4}$ 
A prevalência da $\mathrm{AN}$ varia de $0,5 \%$ a 3,7\%, e a $\mathrm{BN}$ de $1,1 \%$ a 4,2\% no mundo, de acordo com os critérios usados para estabelecer a classificação dos transtornos alimentares (TAs). No Brasil, ainda não é possível encontrar esta informação na literatura. No que diz respeito à predisposição ao desenvolvimento de TAs, o público feminino com idade entre 12 e 25 anos é considerado o grupo com maior susceptibilidade para o surgimento do distúrbio alimentar, pois nesse período geralmente existem modificações na rotina diária, assim como alterações nos hábitos alimentares e na imagem corporal. ${ }^{5}$

Pesquisadores apontam que os estudantes da área da saúde apresentam risco aumentado para o aparecimento de distúrbios alimentares, devido a suas atividades rotineiras. Além disso, para os universitários de Nutrição, um importante fator a considerar é o convívio destes com os alimentos e seu pensamento em relação ao aspecto exterior, atribuindo grande relevância à boa aparência e relacionando-a com seu sucesso profissional. ${ }^{6,7}$

Dessa maneira, salienta-se a importância da realização deste estudo, que aprofunda o conhecimento sobre comportamento alimentar e distorção na imagem corporal, que podem acarretar TAs. Objetivou-se avaliar o estado nutricional agregado à percepção da imagem corporal e ao comportamento alimentar de estudantes do sexo feminino do curso de Nutrição do Instituto Federal de Educação, Ciência e Tecnologia (IFCE), campus Limoeiro do Norte.

\section{Metodologia}

O estudo foi realizado com um delineamento quantitativo e transversal, do tipo descritivo e analítico, e teve como população 130 universitárias do curso de Nutrição. A amostra foi composta por 52 estudantes do sexo feminino que foram escolhidas de forma aleatória. Assim, a amostra foi definida segundo Triola, ${ }^{8}$ que considera nível de confiança de $95 \%$ e erro aceitável de $5 \%$. O estudo foi realizado entre os meses de março e setembro de 2015.

A coleta dos dados foi realizada no Laboratório de Avaliação Nutricional do Instituto Federal de Educação, Ciência e Tecnologia do Ceará (IFCE), campus Limoeiro do Norte, em horários previamente escolhidos e respeitando a disponibilidade das alunas. Foram aplicados três instrumentos autorrespondidos, amplamente utilizados para a detecção de transtornos alimentares (TAs), todos em versões traduzidas para português: o Body Shape Questionnaire - Questionário de Imagem Corporal (BSQ), o Eating Attitudes Test - Teste de Atitudes Alimentares (EAT-26) e o Bulimic Investigatory Test Edinburgh - Teste de Avaliação Bulímica de Edimburgo (Bite).

O BSQ é um questionário que visa avaliar o nível de preocupação com a forma e com peso corporal, além de detectar a desvalorização da aparência física. Esse questionário analisa com precisão a estimativa do tamanho corporal e sentimentos em relação às formas do corpo. É composto por 34 perguntas, com seis possíveis respostas: "nunca" (1 ponto), "raramente" (2 pontos), "às vezes" 
(3 pontos), "frequentemente" (4 pontos), "muito frequentemente" (5 pontos) e "sempre" (6 pontos). A classificação é realizada com base no total de pontos obtidos e demonstra a preocupação com a imagem corporal e a insatisfação com o corpo: normal ( $<70$ pontos): caracterizando ausência de insatisfação com a imagem; leve insatisfação ( $\geq 70$ e $<90$ pontos); moderada insatisfação ( $\geq 90$ e $<110$ pontos); e grave insatisfação ( $\geq 110$ pontos) ${ }^{9}$

O EAT-26 é um questionário utilizado para verificar e identificar atitudes e comportamentos alimentares anormais típicos da AN, sendo composto por 26 perguntas com seis opções de respostas (sempre $=3$ pontos, muitas vezes $=2$ pontos, às vezes, poucas vezes, quase nunca e nunca). Com base na pontuação obtida, os autores que fizeram a tradução colocaram como ponto de corte $\geq 21$ pontos, que indicam o EAT-26 positivo para distúrbios alimentares e risco aumentado para TAs. ${ }^{10}$

O Bite é um instrumento desenvolvido para identificar indivíduos que apresentam compulsão alimentar e que tenham sintomatologias bulímicas. O questionário é composto de 33 perguntas, sendo que a resposta "sim" representa a presença do sintoma, valendo um ponto, enquanto a resposta "não" significa a ausência (0 ponto). Nas questões 1, 13, 21, 23 e 31, pontua-se inversamente. Sua classificação ocorreu da seguinte maneira: baixo (> 10 pontos): sem sintomas de BN; médio (10 a 19): comportamento alimentar não usual; e alto (> 20 pontos): com sintomas de BN e grande possibilidade de preencher os critérios para o diagnóstico. ${ }^{11}$

Em relação à escala de silhuetas, utilizou-se uma versão criada e validada por kakeshita ${ }^{12}$ que visa avaliar e auxiliar na verificação da prevalência da insatisfação corporal dos participantes. A escala é composta por um conjunto de 15 silhuetas, onde cada silhueta tem um IMC médio correspondente, que varia de 12,5 a $47,5 \mathrm{Kg} / \mathrm{m}^{2}$. Para a aplicação do teste, foi solicitado que cada pessoa escolhesse primeiramente uma silhueta que melhor representasse sua forma física atual (IMC atual), e depois que indicasse a silhueta que gostaria de possuir (IMC desejado). A diferença entre o IMC atual e IMC desejado permitiu avaliar a percepção corporal dos participantes.

Além disso, foi realizada a aferição do peso e altura para cálculo do índice de massa corporal (IMC aferido), que foi a razão entre a massa corporal $(\mathrm{kg})$ e a estatura elevada ao quadrado $\left(\mathrm{m}^{2}\right),{ }^{13}$ com o objetivo de compará-lo aos valores encontrados na Escala de Silhuetas. Os participantes do estudo foram pesados em uma balança antropométrica digital Toledo ${ }^{\circledR}$, com capacidade de $200 \mathrm{~kg}$ e intervalo de $100 \mathrm{~g}$. A estatura foi aferida com um estadiômetro Professional Sanny ${ }^{\circledR}$, com capacidade de 40 a $210 \mathrm{~cm}$. A classificação do IMC ocorreu com base nos critérios da Organização Mundial da Saúde. ${ }^{14}$

Foram utilizadas análises descritivas de média \pm desvio padrão e frequências simples e relativas. Para a análise de diferenças estatísticas, utilizou-se a análise de variância (Anova), e o nível de significância entre os grupos foi determinado pelo teste de Turkey. Para a análise dos dados, foi utilizado o programa GraphPad Prism, versão 5.03 ${ }^{\circledR}$. 
A presente pesquisa foi avaliada e aprovada pelo Comitê de Ética em Pesquisa da Escola de Saúde Pública do Ceará, com parecer n⿳0 946.036. Os participantes assinaram o Termo de Consentimento Livre e Esclarecido (TCLE), onde foram expressas, de maneira clara, as informações e orientações sobre a pesquisa. Assim, todos os procedimentos que foram realizados neste estudo estão em acordo com as determinações institucionais e a Resolução no 466/2012 do Conselho Nacional de Saúde, que trata das diretrizes e normas regulamentadoras de pesquisas envolvendo seres humanos.

\section{Resultados}

A amostra foi composta de 52 participantes com médias \pm desvio padrão (DP) para idade de $23 \pm 4$ anos; IMC de 23,39 \pm 4,01 kg/m²; altura aferida de $158,0 \pm 5 \mathrm{~cm}$ e peso aferido de 58,59 $\pm 10,91 \mathrm{~kg}$. A tabela 1 mostra os dados referentes a média, desvio padrão e porcentagens do IMC por classificação.

No que diz respeito aos resultados dos questionários, a média geral de pontuação para o BSQ foi 73,94 \pm 32,91 pontos; para EAT-26, foi 10,17 \pm 7,55 pontos; e para Bite, foi 4,25 \pm 3,65 pontos. A tabela 2 evidencia a prevalência de comportamentos alimentares inadequados por meio dos resultados obtidos pelo EAT-26 e pelo Bite e a insatisfação com a imagem corporal avaliada pelo BSQ em universitárias de Nutrição. Observa-se, na tabela 3, a relação entre a classificação do IMC com os resultados encontrados (média, desvio padrão e nível de significância) com os questionários EAT-26, Bite e BSQ.

No que se refere à Escala de Silhueta, os dados expressam que as participantes classificadas como desnutridas apresentaram, em totalidade, insatisfação por magreza; as estudantes com sobrepeso e obesidade apresentaram em geral insatisfação por excesso de peso; e as eutróficas, em sua maioria (72,7\%), apresentaram-se insatisfeitas por excesso de peso (tabela 4).

Tabela 1. Classificação do IMC em universitárias de Nutrição. Limoeiro do Norte, Ceará, 2015.

\begin{tabular}{lccc}
\hline \multicolumn{1}{c}{ IMC $\left(\mathrm{kg} / \mathrm{m}^{2}\right)$} & Média & DP & $\%$ \\
\hline Baixo-Peso & 18,04 & $\pm 0,6$ & 5,77 \\
Normal/Eutrófico & 21,56 & $\pm 1,8$ & 63,46 \\
Sobrepeso & 27,19 & $\pm 1,4$ & 26,92 \\
Obesidade & 34,89 & $\pm 5,1$ & 3,85 \\
\hline
\end{tabular}

Fonte: (OMS, 1995). ${ }^{14}$ 
Tabela 2. Prevalência da insatisfação com a imagem corporal e comportamentos alimentares inadequados em universitárias de Nutrição. Limoeiro do Norte, Ceará, 2015.

\begin{tabular}{lcc}
\hline \multicolumn{1}{c}{ Escala (pontuação) } & $\%$ & IC 95\% \\
\hline BSQ insatisfação grave & 15,38 & $12,9-17,8$ \\
BSQ insatisfação moderada & 9,61 & $5,8-13,4$ \\
BSQ insatisfação leve & 21,15 & $13,6-28,7$ \\
BSQ satisfação & 53,85 & $39,6-68,2$ \\
EAT positivo & 9,62 & $3,0-16,3$ \\
EAT negativo & 90,38 & $71,0-110,1$ \\
Bite Alto & 0 & ---- \\
Bite Médio & 15,38 & $14,4-16,4$ \\
Bite Baixo & 84,62 & $67,1-102,1$ \\
\hline
\end{tabular}

IC = Intervalo de Confiança, com confiança estabelecida em $95 \%$ dos casos.

Tabela 3. Relação entre a classificação do IMC com a pontuação média do EAT-26, do Bite e do BSQ em universitárias de Nutrição. Limoeiro do Norte, Ceará, 2015.

\begin{tabular}{lccc}
\hline IMC $\left(\mathrm{Kg} / \mathrm{m}^{2}\right)$ & & EAT-26 & $\mathrm{P}$ \\
\cline { 2 - 4 } Baixo-Peso & Média & $\mathrm{DP}$ & $*$ \\
Normal/Eutrófico & 10,33 & 0,6 & $*$ \\
Sobrepeso & 7,84 & 5,2 & $*$ \\
Obesidade & 15,0 & 9,6 & 0,257 \\
\cline { 2 - 4 } & 14,5 & 17,7 & $\mathrm{P}$ \\
\cline { 2 - 4 } & & $\mathrm{BITE}$ & 0,0048 \\
Baixo-Peso & Média & $\mathrm{DP}$ & $*$ \\
Normal/Eutrófico & 3,3 & 1,6 & $*$ \\
Sobrepeso & 3,3 & 3,2 & $*$ \\
Obesidade & 5,9 & 4,0 & $\mathrm{P}$ \\
& 8,5 & 3,5 & 0,0178 \\
\cline { 2 - 4 } & & $\mathrm{BSQ}$ & $*$ \\
Baixo-Peso & Média & $\mathrm{DP}$ & $*$ \\
Normal/Eutrófico & 61,3 & 19,3 & 0,0128 \\
Sobrepeso & 62,5 & 22,0 & 29,3 \\
Obesidade & 96,4 & 19,1 & \\
\hline & 157,5 & & \\
\hline
\end{tabular}


Tabela 4. Relação entre a classificação do IMC aferido com os resultados encontrados na Escala de Silhuetas em universitárias de Nutrição. Limoeiro do Norte, Ceará, 2015.

\begin{tabular}{|c|c|c|c|c|c|c|c|}
\hline \multirow[t]{2}{*}{$\begin{array}{l}\text { IMC aferido } \\
\qquad\left(\mathrm{kg} / \mathrm{m}^{2}\right)\end{array}$} & \multicolumn{2}{|c|}{ IMC atual } & \multicolumn{2}{|c|}{$\begin{array}{c}\text { IMC } \\
\text { desejado }\end{array}$} & \multirow{2}{*}{$\begin{array}{c}\begin{array}{c}\text { Insatisfeito } \\
\text { por magreza }\end{array} \\
\%\end{array}$} & \multirow{2}{*}{$\begin{array}{l}\begin{array}{l}\text { Insatisfeito por } \\
\text { excesso de peso }\end{array} \\
\%\end{array}$} & \multirow[t]{2}{*}{$\mathrm{P}$} \\
\hline & M & $\mathrm{D}$ & M & $\mathrm{D}$ & & & \\
\hline Desnutrido & 19,7 & 2,5 & 25,8 & 3,8 & 100 & 0 & 0,0251 \\
\hline $\begin{array}{l}\text { Normal/ } \\
\text { Eutrófico }\end{array}$ & 22,6 & 5,2 & 23,6 & 3,1 & 27,3 & 72,7 & $*$ \\
\hline Sobrepeso & 32,5 & 4,2 & 26,6 & 3,5 & 0 & 100 & 0,5641 \\
\hline Obesidade & 42,5 & 3,5 & 24,6 & 3,7 & 0 & 100 & 0,3546 \\
\hline
\end{tabular}

* $\mathrm{P}<0,001$

\section{Discussão}

A prevalência de risco do EAT-26 foi de 9,62\%, e do Bite foi de 15,38\%, demonstrando a presença de hábitos alimentares inadequados pelas acadêmicas de Nutrição. Os dados encontrados não indicam casos severos para o EAT-26 e para o Bite. Os achados na literatura, em geral, evidenciam casos moderados, segundo as classificações dos questionários. Assim, é importante ressaltar uma possível evolução desses quadros para grau mais elevado, contribuindo para o aparecimento de TAs. ${ }^{15,16}$

Na comparação com outros estudos realizados com estudantes de Nutrição que realizaram o EAT-26, a prevalência de comportamento de risco para AN do presente estudo foi similar aos 11,8\% encontrados no estudo com alunas da Nutrição em Porto Alegre-RS. ${ }^{17}$ Entretanto, os valores deste estudo foram menores que os 20,2\% encontrados entre alunas de Nutrição de uma instituição privada de ensino superior do Centro-Oeste do país, ${ }^{7}$ e que os 21,03\% encontrados em alunas de Nutrição da Faculdade Federal de Juiz de Fora-MG. Sugere-se que esta prevalência pode ser esclarecida pela relação entre aparência física dentro do padrão estabelecido pela sociedade, que enaltece a magreza para público feminino, como os conteúdos adquiridos na graduação de Nutrição. ${ }^{18}$

Com relação à BN apontada pelo Bite no atual estudo, não foram achados dados que indicassem a presença de sintomas característicos desse distúrbio, mas se encontrou um percentual de 15,38\%, indicando padrão alimentar não usual. Embora esse percentual não seja baixo, ainda está abaixo dos 20,9\% encontrados entre os universitários da Universidade Federal de Santa Catarina ${ }^{19}$ e os 24,32\% relacionados aos estudantes de Nutrição do Centro Universitário do Leste de Minas Gerais. ${ }^{20}$

No confronto com pesquisas realizadas com universitários de outras áreas da saúde, podemos colocar que a prevalência do atual estudo, no que diz respeito ao EAT-26, foi maior que os 8,9\% 
encontrados nas estudantes de Psicologia e menor que os 12,2\% das universitárias de Enfermagem de estudo realizado no Centro-Oeste brasileiro; ${ }^{7}$ e ainda, que os $11,1 \%$ de estudantes de Educação Física de uma Universidade de Minas Gerais. ${ }^{20}$ Em relação ao Bite, no presente estudo o percentual encontrado foi de $15,38 \%$ de estudantes que apresentaram padrão alimentar não usual, sendo este maior que os 13,88\% encontrados em universitários de Educação Física ${ }^{20}$ e menor que 47,2\% apresentados em outro estudo com 72 estudantes de Enfermagem e Fisioterapia. ${ }^{21}$

Em relação ao BSQ, a prevalência de insatisfação com a imagem corporal foi de 46,1\%, contemplando as classes leve $(21,15 \%)$, moderada $(9,61 \%)$ e grave $(15,38 \%)$. Destaca-se ainda o fato de que 72,7\% das eutróficas, segundo a Escala de Silhueta, apresentaram-se insatisfeitas por excesso de peso, mostrando assim o desejo de perda de peso. Contudo, uma maioria de 63,5\% das estudantes apresentou IMC dentro dos padrões de eutrofia, sugerindo assim um episódio clássico de superestimação de peso corporal por parte da população em estudo. Na presente pesquisa, quando relacionamos o estado nutricional com a insatisfação corporal pelo BSQ, foi possível verificar que a classificação do IMC está significativa e diretamente associada à pontuação obtida no BSQ.

Na comparação com outros estudos que utilizaram o BSQ, para avaliar o nível de insatisfação com a imagem corporal, identificou-se resultado similar, em que 47,3\% dos estudantes de diferentes cursos de graduação na área da saúde de uma Universidade de Santa Catarina encontravam-se insatisfeitos com a imagem corporal. ${ }^{22}$

Com o intuito de evidenciar que esses TAs estão presentes também em alunas de nível médio, estudo foi realizado com este público, de escola pública de Florianópolis-SC, apresentando resultado de $18,8 \%$, inferior ao encontrado na atual investigação. Esta diferença pode ser atribuída aos diferentes contextos em que as pesquisas foram realizadas. ${ }^{23}$ Um estudo com 175 alunas do curso de Nutrição da Universidade Federal de Ouro Preto apontou que 36,6\% apresentavam algum nível de distorção na imagem do corpo. ${ }^{24}$

No entanto, em pesquisa realizada por Moreira e colaboradores,${ }^{25} \mathrm{com}$ universitários do curso de Medicina da Bahia, foi encontrado que $50 \%$ apresentavam-se insatisfeitos com a imagem, sendo este resultado superior ao achado neste estudo. Um resultado comum entre as duas pesquisas foi que os valores da pontuação do BSQ foram maiores nas universitárias com IMC eutrófico do que nas universitárias com IMC baixo-peso. ${ }^{25}$

Em relação à Escala de Silhueta, os resultados encontrados mostraram-se similares, com elevados valores de descontentamento com a imagem corporal. No estudo de Miranda e colaboradores, ${ }^{26}$ foi encontrado que 76,6\% dos universitários apresentavam-se insatisfeitos, sendo que $61,7 \%$ desejavam ter uma silhueta menor. Em investigação científica realizada por Martins e colaboradores ${ }^{27}$ com 865 universitários de diversos cursos de graduação, 77,9\% apresentam-se insatisfeitos, sendo que no público feminino $62,4 \%$ estavam insatisfeitos por excesso de peso e $15,5 \%$ por magreza. ${ }^{26}$ 
Portanto, tais resultados evidenciam que os jovens apresentam forte preocupação com a imagem corporal, e quando esse estado de insatisfação toma proporções exacerbadas, o resultado pode ser o desenvolvimento de transtornos alimentares diversos.

A população-alvo deste estudo apresentou um problema frequente, a insatisfação e a distorção na imagem corporal, que leva a uma superestimação do peso, acontecimento que foi relatado em diversos estudos com universitários. Dessa maneira, é possível observar que o estado nutricional, segundo o IMC, mostra associação com a imagem corporal, o que demostra a busca das estudantes pela adequação a esse corpo tido como "ideal". As motivações podem variar desde a busca por hábitos saudáveis quanto a adaptação aos modelos sociais de beleza, até uma insatisfação com a imagem corporal de forma doentia, assim como o desenvolvimento de hábitos alimentares inadequados - principais fatores de risco para o desenvolvimento de Tas. ${ }^{22,24}$

Alguns estudos apontam uma conjectura da percepção do peso corporal sobrepondo-se à classificação do IMC, isto é, a forma como a pessoa se observa é mais decisiva para as mudanças nos hábitos alimentares que a própria composição do corpo. A presença de insatisfação com a imagem, principalmente no público feminino, acarreta um comportamento alimentar e formas de redução de peso inadequadas, por meio do uso de laxantes, diuréticos, vômitos induzidos, atividade física em excesso, entre outras. Assim é importante que os profissionais da saúde levem em consideração estas ocorrências, para que possam fazer uma detecção precoce do surgimento dos TAs, seguindo com a prevenção da gravidade dos sintomas e o tratamento dos episódios. ${ }^{20,24}$

O presente estudo apresenta algumas limitações metodológicas, dentre as quais pode-se citar: o uso de questionários que apresentam apenas características dos sintomas dos TAs, pois os mesmos são insuficientes para confirmar o diagnóstico e o fato de ser um estudo transversal, visto que não se observou uma relação de tempo entre as variáveis, o que é possível somente nas pesquisas longitudinais, com acompanhamento contínuo. ${ }^{22}$

Neste contexto, pode-se indicar que o desenvolvimento dos TAs e de seus fatores de risco possui relação importante com os padrões de beleza, com a imagem corporal, com o pensamento em relação ao alimento e com o próprio corpo.

\section{Conclusão}

O presente estudo mostrou menor prevalência em relação aos resultados dos questionários EAT-26 e Bite. Esses instrumentos indicam casos de comportamento alimentar inadequado leves ou moderados, podendo assim ocorrer a evolução para casos mais severos. No entanto, a pesquisa apresentou elevada prevalência de insatisfação com a imagem corporal, resultado expresso por dois instrumentos, o BSQ e a Escala de Silhueta. 
Portanto, é importante que haja a elaboração de estratégias para prevenção dos transtornos alimentares (TAs), visando a mudanças positivas no comportamento alimentar e na forma de percepção da aparência física da população em estudo, visto que se trata de um grupo de risco para desenvolvimento de TAs.

\section{Colaboradores}

Maia RGL; Fiorio BC; de Almeida JZ e da Silva, FR participaram de todas as etapas, desde a concepção do estudo até a revisão da versão final do artigo.

Conflito de interesses: os autores declaram não haver conflito de interesses.

\section{Referências}

1. Phillipi ST, Alvarenga M, Scagliusi FB. Nutrição e transtornos alimentares: avaliação e tratamento. São Paulo: Manole; 2011.

2. Cordás TA, Salzano FT, Rios, SR. Os transtornos alimentares e a evolução no diagnóstico e tratamento. In: Philippi ST, Alvarenga M. Transtornos alimentares: uma visão nutricional. São Paulo: Manole; 2004. p. 39-62.

3. Abreu CN, Cangelli-Filho, R. Anorexia nervosa e bulimia nervosa: a abordagem cognitivoconstrutivista de psicoterapia. Psicologia: teoria e prática. 2005; 7(1):153-165.

4. Ferreira CP. Disfonia e bulimia: avaliação dos sintomas e sinais vocais e laríngeos. Rev Soc Bras Fonoaudiol. 2008; 14(2):177-185.

5. Oliveira-Cardoso EA, Zuben BVV, Santos MA. Qualidade de vida de pacientes com anorexia e bulimia nervosa. DEMETRA: Alimentação, Nutrição \& Saúde. 2014; 9(Supl. 1):329-340.

6. Fiates GMR, Salles RK. Fatores de risco para o desenvolvimento de distúrbios alimentares: um estudo em universitárias. Rev Nutr. 2001; 14(Supl.):3-6.

7. Souza AA, Souza JC, Hirai ES, Luciano HA, Souza N. Estudo sobre a Anorexia e Bulimia Nervosa em universitárias. Psic: Teor e Pesq. 2011; 27(2):195-198.

8. Triola MF. Introdução à estatística. 7 ed. Rio de Janeiro: Livros Técnicos e Científicos; 1999.

9. Cordás TA, Castilho S. Imagem corporal nos transtornos alimentares. instrumento de avaliação. Psiquiatr Biol. 1994; 2(1):17-21.

10. Nunes MA, Camey S, Olinto MTA, Mari JJ. The validity and 4-year test-retest reliability of the Brazilian version of the Eating Attitudes Test-26. Braz J Med Biol Res. 2005; 38(16):55-62.

11. Cordás TA, Hochgraf PB. O BITE: instrumento para avaliação da bulimia nervosa versão para o português. J Bras Psiquiatr. 1993; 3(42):4-141.

12. Kakeshita IS. Adapatação e validação de escalas de silhuetas para crianças e adultos brasileiros [tese]. [São Paulo]: Universidade de São Paulo; 2008. 
13. Cervi A, Franceschini SCC, Priore SE. Análise crítica do uso de índice de massa corporal para idosos. Rev Nutr. 2005; 18(6):765-775.

14. World Health Organization. Physical status: the use and interpretation of anthoropometry. Report of a WHO Expert Committee. Geneva: WHO; 1995. Technical report series, 854.

15. Fichter MM, Quadflieg N, Hedlund S. Twelve-year course and outcome predictors of anorexia nervosa. Int J Eat Disod. 2006; 39(2):87-100.

16. Souto S, Ferro-Bucher JSN. Práticas indiscriminadas de dietas de emagrecimento e o desenvolvimento de transtornos alimentares. Rev Nutr. 2006; 19(6):693-704.

17. Garcia CA, Castro TG, Soares, RM. Comportamento alimentar e imagem corporal entre estudantes de nutrição de uma universidade pública de Porto Alegre - RS. Revista HCPA. 2010; 30(3):219-224.

18. Ferreira KLA. Estado nutricional, transtornos alimentares e consumo de álcool entre estudantes do curso de Nutrição de uma Instituição Federal. Juiz de Fora [Trabalho de Conclusão de Curso]. [Juiz de Fora]: Universidade Federal de Juiz de Fora; 2013.

19. Cenci M, Peres KG, Vasconcelos FA. Prevalence of bulimic behavior and associated factors in undergraduate female students. Rev Psiquiatr Clin. 2009; 36(3):83-88.

20. Santos RCE, Gonçalves MA, Stracieri APM. Fatores de risco para anorexia e bulimia nervosas em universitárias dos cursos de Nutrição e Educação Física do Centro Universitário do Leste de Minas Gerais. Nutrir Gerais 2010; 4(6):580-592.

21. Umebara LM; Virote W. Comportamento de risco para transtornos alimentares em universitários. VI Fórum Científico FAP; 2012; Apucarama. Disponível em: http://www.fap.com.br/forum_2012/ forum/pdf/Saude/Comunicacao_Oral/ResSauCO03.pdf

22. Costa LCF, Vasconcelos FAG. Influência de fatores socioeconômicos, comportamentais e nutricionais na Insatisfação com a imagem corporal de universitários. Rev Bras Epidemiol. 2010; 13(4):665-676.

23. Alves E, Vasconcelos FAG, Caldo MCM, Neves J. Prevalência de sintomas de anorexia nervosa e insatisfação com a imagem corporal em adolescentes do sexo feminino do município de Florianópolis, Santa Catarina, Brasil. Cad Saúde Pública. 2008; 24(3):503-512.

24. Silva JD, Silva ABJ; Oliveira AVK, Nemer ASA. Influência do estado nutricional no risco para transtornos alimentares em estudantes de nutrição. Ciênc Saúde Coletiva. 2012; 17(12):169-174.

25. Moreira LAC, Azevedo ABG, Queiroz D, Moura L, Santo DE, Cruz R, et al. Body image in a sample of undergraduate medical students from Salvador, Bahia, Brazil. J Bras Psiquiatr. 2005; 54(4):294-297.

26. Miranda VPN, Filgueiras JF, Neves CM, Texeira PC; Ferreira MAC. Insatisfação corporal em universitárias de diferentes áreas de conhecimento. J Bras Psiquiat. 2012; 61(1):25-32.

27. Martins CR, Pelegrini A, Matheus SC, Petroski EL. Body image dissatisfaction and its relationship with nutritional status, body fat, and anorexia and bulimia symptoms in adolescents. Rev Psiquiatr. 2010; 32(1):19-23.

Recebido: 02 de outubro, 2017

Revisado: 01 de fevereiro, 2018

Aceito: 19 de fevereiro, 2018 
